\title{
Localized surface plasmon resonance in Au nanoprisms on glass substrates
}

\author{
O.G. Lopatynska ${ }^{1}$, A.M. Lopatynskyi ${ }^{2}$, T.I. Borodinova $^{3}$, V.I. Chegel $^{2}$, L.V. Poperenko ${ }^{1}$ \\ ${ }^{I}$ Taras Shevchenko National University of Kyiv, \\ 64/13, Volodymyrska Str., 01601 Kyiv, Ukraine \\ ${ }^{2} V$. Lashkaryov Institute of Semiconductor Physics, NAS of Ukraine, \\ 41, prospect Nauki Ave., 03680 Kyiv, Ukraine \\ ${ }^{3}$ F. Ovcharenko Institute of Biocolloid Chemistry, NAS of Ukraine, \\ 42, Acad. Vernadsky Blvd., 03680 Kyiv, Ukraine \\ Phone: +38(050)824-42-03, e-mail: olga_lopatynska@ukr.net,lop2000@ukr.net, borodinova@ua.fm, \\ vche111@yahoo.com,plv@univ.kiev.ua
}

\begin{abstract}
Metal nanocrystals are actual objects for the modern biophysics mainly because of their usage in sensors based on localized surface plasmon resonance (LSPR) and as active substrates for surface-enhanced spectroscopies. This work deals with the experimental and theoretical investigation of optical properties of trigonal and hexagonal $\mathrm{Au}$ nanoprisms deposited on the glass substrates. It was confirmed for the studied structures that the LSPR spectra depend on the crystals shape and size. Theoretical modeling the optical properties of plasmon-supporting nanoprisms was performed using the finite-difference time-domain method. The experimentally obtained and theoretically modeled LSPR spectral positions were found to be different, which can be attributed to a high spread of nanoprism shapes and sizes in the same sample and to nanocrystals aggregation effect confirmed by microscopy data. Additionally, the distributions of the electric field in the vicinity of nanoprisms under the LSPR conditions were simulated, and a strong field intensity enhancement at the corners of the prisms was demonstrated, which implies the promising application of such plasmonic nanostructures for surfaceenhanced spectroscopy.
\end{abstract}

Keywords: localized surface plasmon resonance, Au nanoprism, finite-difference timedomain method.

Manuscript received 12.05.15; revised version received 03.09.15; accepted for publication 28.10.15; published online 03.12.15.

\section{Introduction}

Localized surface plasmon resonance (LSPR) phenomenon plays a significant role in the modern nanobiophysics, especially, due to its promising applications in biosensing [1], surface-enhanced infrared absorption (SEIRA) [2] and surface-enhanced Raman spectroscopy (SERS) [3,4]. It is known that the spectral position of LSPR and accompanying local electric field enhance- ment strongly depend on the shape and size of the nanoparticles [5], as well as on the interparticle distance and the level of ordering in the surface-bound nanoparticle arrays [6]. Therefore, depending on the plasmon resonance parameters needed, noble metal nanostructures of different sizes and morphologies are used as LSPR-supporting materials. For example, spherical Au nanoparticles of different sizes has been exploited for the response enhancement of the surface 
plasmon resonance sensing system, and the optimal nanoparticle diameter of $40 \mathrm{~nm}$ was reported to give the best amplification [7]. Henry et al. [8] investigated Ag nanocube ensembles in water and individual Ag nanocubes on the glass substrate and found striking differences in respective LSPR spectra due to an expressed multimodal LSPR excitation in the latter case. Size- and shape-dependent LSPR properties of noble metal nanodisks [9], nanorods [10] and nanopyramids [11] have been also demonstrated. From the theoretical point of view, optical properties of the plasmonic nanoparticles are studied by means of analytical and numerical methods to simulate interaction of electromagnetic waves with nanoparticles. One of the most common analytical techniques is Mie scattering approach [12], which is applicable only for the spherical or ellipsoidal particles, while for the nonspherical nanostructures numerical methods are usually preferred. One of them is the finitedifference time-domain method (FDTD) [13], which advantages and disadvantages have been reported earlier [14].

In this work, we experimentally investigate the LSPR properties of chemically synthesized trigonal and hexagonal $\mathrm{Au}$ nanoprisms deposited on the glass substrates and theoretically simulate LSPR spectra of these nanostructures by means of the finite-difference time-domain method. The analysis of local electric field distribution near the surface of trigonal and hexagonal $\mathrm{Au}$ nanoprisms with different lateral dimensions is also presented.

\section{Microscopy of trigonal and hexagonal Au nanoprisms deposited on glass substrates}

Fabrication technology of the studied samples was described in the previous work [15]. Briefly, after synthesis $\mathrm{Au}$ nanocrystals were separated from the disperse medium by centrifugation (centrifuge OPN-8, $800 \mathrm{~min}^{-1}, 60 \mathrm{~min}$ ), rinsed with distilled water, precipitate was resuspended in the distilled water. The crystals suspension washed from the stabilizer was dropcasted on the glass substrates and dried at the room temperature. According to the protocol, plain $\mathrm{Au}$ nanoprisms have the shapes of regular triangle, hexagon and truncated triangle with the average linear size of $100 \mathrm{~nm}$ and height of $60 \mathrm{~nm}$.

The microphotographs of the synthesized $\mathrm{Au}$ nanocrystals (Fig. 1a, b), dark field image of large $\mathrm{Au}$ crystal (Fig. 1c) and electron diffraction pattern of the latter crystal (Fig. 1d) confirming the nanoprism monocrystallinity are presented in Fig. 1. The microphotographs were obtained using transmission electron microscope JEM 100CX (JEOL, Japan) with the accelerating voltage of $U=100 \mathrm{kV}$. Optical microscopy of the samples was performed using OLYMPUS GX-41 microscope (Melitek-Ukraine, Ukraine) with the $\times 100$ objective in the light field mode. Optical microscopy revealed a huge spread of nanoprism sizes and the effect of nanocrystals aggregation (Fig. 1e, f).
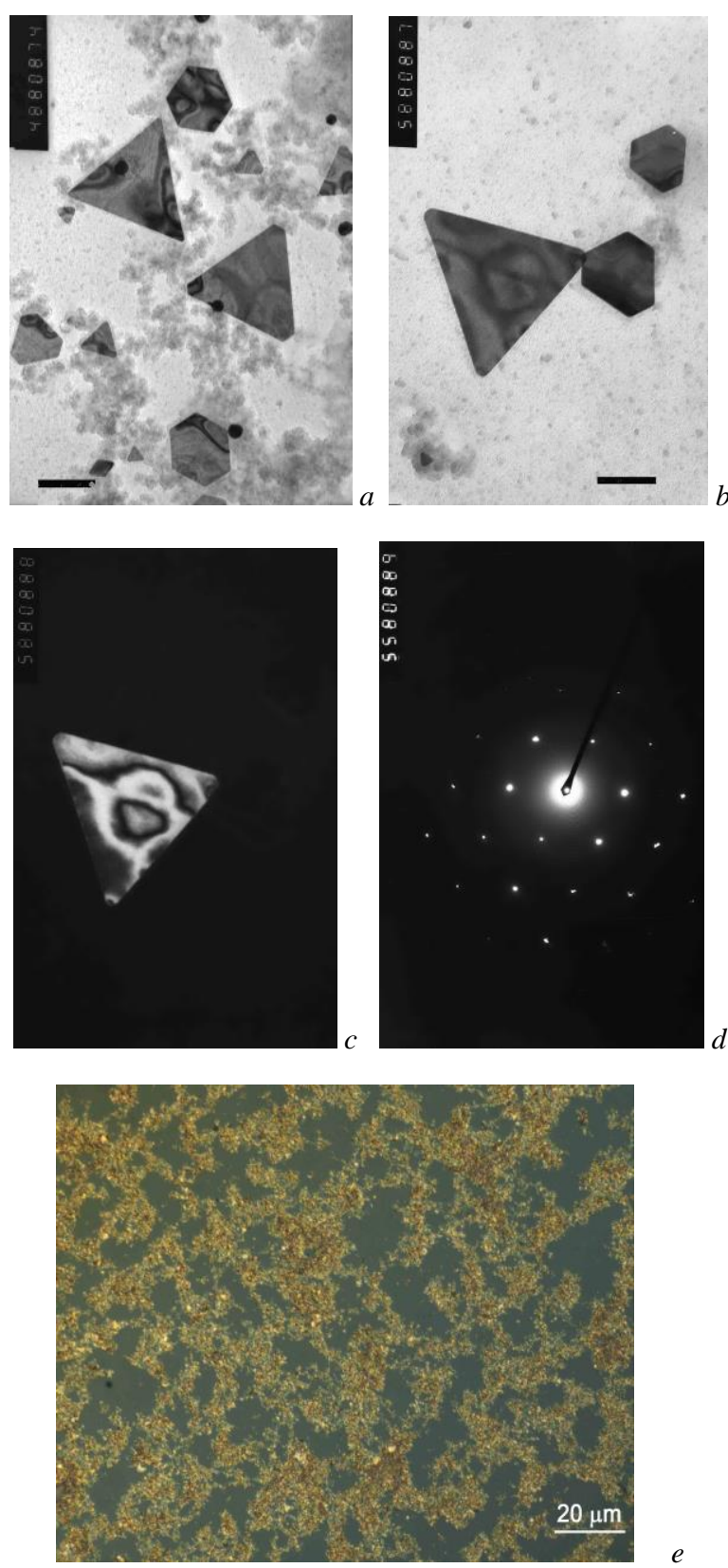

$e$

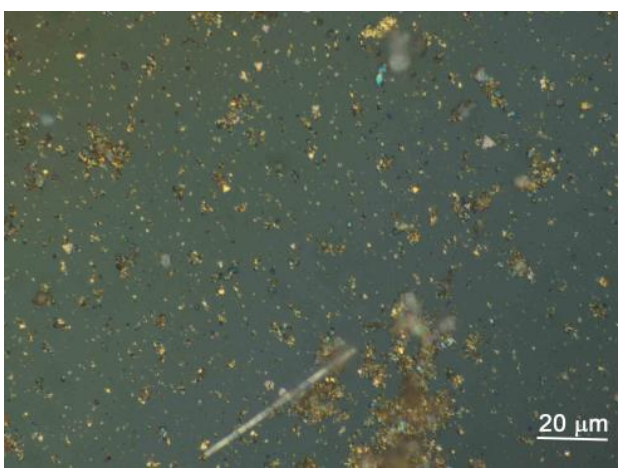

$f$

Fig. 1. $(a, b)$ TEM microphotographs of Au nanoprisms (scale bar is $50 \mathrm{~nm}$ ); (c) dark field image of large Au crystal in (b); (d) electron diffraction pattern of large Au crystal in (b); (e, f) optical microscopy images of Au nanoprisms deposited on the glass substrates (magnification is $\times 1000$ ).

\section{(C) 2015, V. Lashkaryov Institute of Semiconductor Physics, National Academy of Sciences of Ukraine}




\section{LSPR in the trigonal and hexagonal Au nanoprisms deposited on glass substrates}

Light extinction spectra of the samples were measured using the LSPR spectrometer "Nanoplasmon-003" (V. Lashkaryov Institute of Semiconductor Physics, NAS of Ukraine).

Light absorbance measurements have shown the existence of a broad complex absorption band within the spectral range $500 \ldots 1000 \mathrm{~nm}$ with a distinct peak in the visible region at $591 \mathrm{~nm}$ (Fig. 2). The appearance of this absorption band can be attributed to the LSPR excitation in surface-bound $\mathrm{Au}$ nanoparticles, while its complex form and broadening are possibly observed due to distribution of shapes and sizes of Au nanoparticles with several dominating geometrical configurations.

To investigate theoretically the LSPR spectra of trigonal and hexagonal Au nanoprisms, we modelled the light extinction cross-section spectra for trigonal $\mathrm{Au}$ nanoprisms with side dimensions of $100 \mathrm{~nm}$ (model 1, Fig. 3a), $25 \mathrm{~nm}$ (model 1, Fig. 3b), hexagonal $\mathrm{Au}$ nanoprisms with side dimensions of $33 \mathrm{~nm}$ (model 2, Fig. 3a) and $8 \mathrm{~nm}$ (model 2, Fig. 3b), which were obtained by truncation of trigonal nanoprisms to get regular hexagonal nanoprism. Additionally, calculations were performed for the combination of trigonal $\mathrm{Au}$ nanoprism with side dimension of $25 \mathrm{~nm}$ and hexagonal $\mathrm{Au}$ nanoprism with side dimension of $8 \mathrm{~nm}$ with interparticle distance of $30 \mathrm{~nm}$ (model 3, Fig. 3b), which models the influence of nanoprism shape distribution on the light extinction spectrum. In all the considered models, nanoprisms were located on glass substrates and the height of the nanoprisms was equal to $60 \mathrm{~nm}$. All calculations were performed using the commercial FDTD package (Lumerical FDTD Solutions, trial version, Lumerical Solutions, Inc., Canada). The experimental absorbance spectrum and the theoretical extinction cross-section spectra corresponding to the abovementioned models are presented in Fig. 3.

As it can be seen from Fig. 3, simulated and experimental LSPR spectral positions and shapes are different. This can be explained by several factors related to the fact that theoretical spectra correspond to individual nanoparticles and experimental spectrum characterizes the whole sample. First, the interaction between the nanoparticles was not taken into account. Second, the effect of nanoprisms aggregation was present in the sample. Third, there were a broad distribution of nanoparticle sizes and several different nanoparticle shapes in the same sample. However, it should be noted that the best agreement with the experimental spectrum was produced by the model 2 (Fig. 3a). Therefore, we can conclude that the experimentally observed LSPR response results mainly from the $100 \mathrm{~nm}$ hexagonal $\mathrm{Au}$ nanoprisms, but the contribution of $\mathrm{Au}$ nanoprisms with other shapes and sizes is still significant.

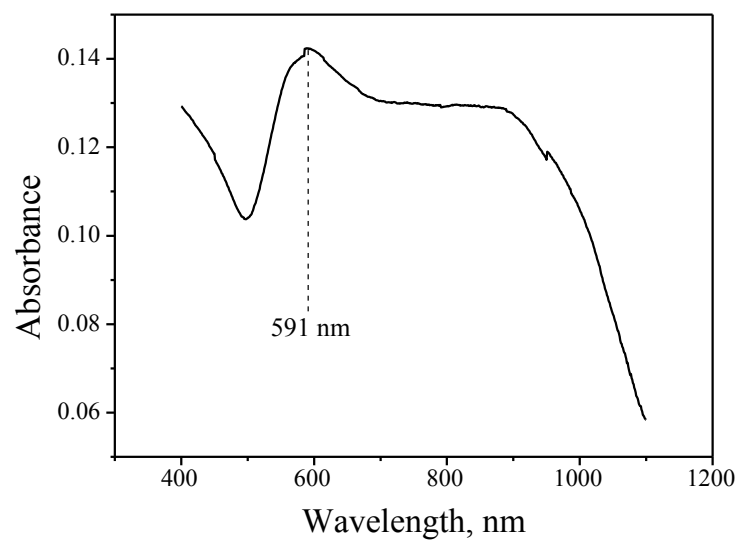

Fig. 2. Experimental light absorbance spectrum of $\mathrm{Au}$ nanoprisms deposited on the glass substrate.

(a)

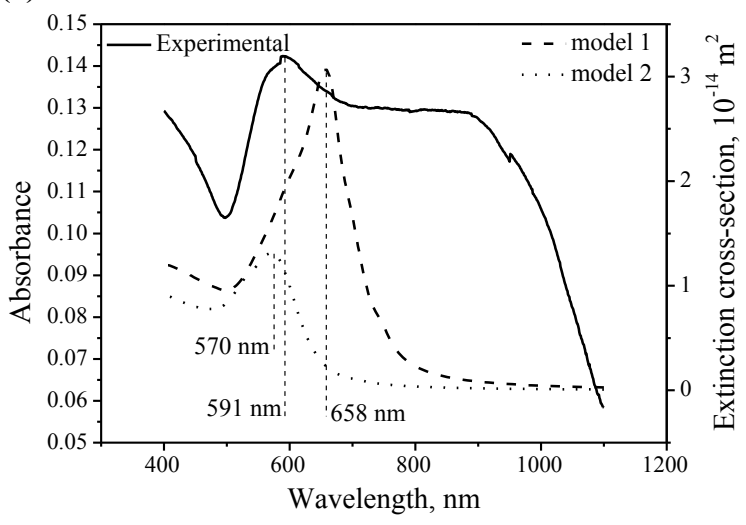

(b)

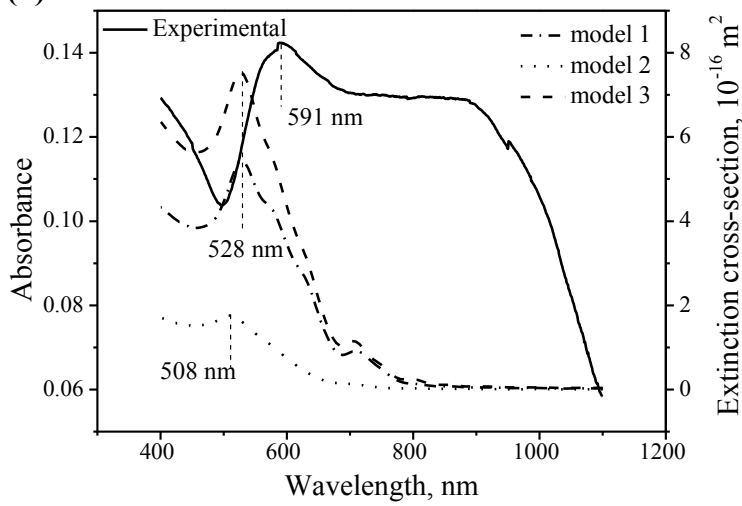

Fig. 3. The experimental absorbance spectrum of the $A u$ nanoprisms on the glass substrate and the theoretical extinction cross-section spectra of (a) model 1 - trigonal Au nanoprism with side dimension of $100 \mathrm{~nm}$; model 2 - hexagonal Au nanoprism with side dimension of $33 \mathrm{~nm}$; (b) model 1 trigonal Au nanoprism with side dimension of $25 \mathrm{~nm}$; model 2 - hexagonal Au nanoprism with the side dimension of $8 \mathrm{~nm}$; model 3 - combination of trigonal and hexagonal $\mathrm{Au}$ nanoprisms with side dimensions of $25 \mathrm{~nm}$ and $8 \mathrm{~nm}$, respectively, and interparticle distance of $30 \mathrm{~nm}$. All nanoprisms were located on the glass substrates and the height of nanoprisms was equal to $60 \mathrm{~nm}$. 

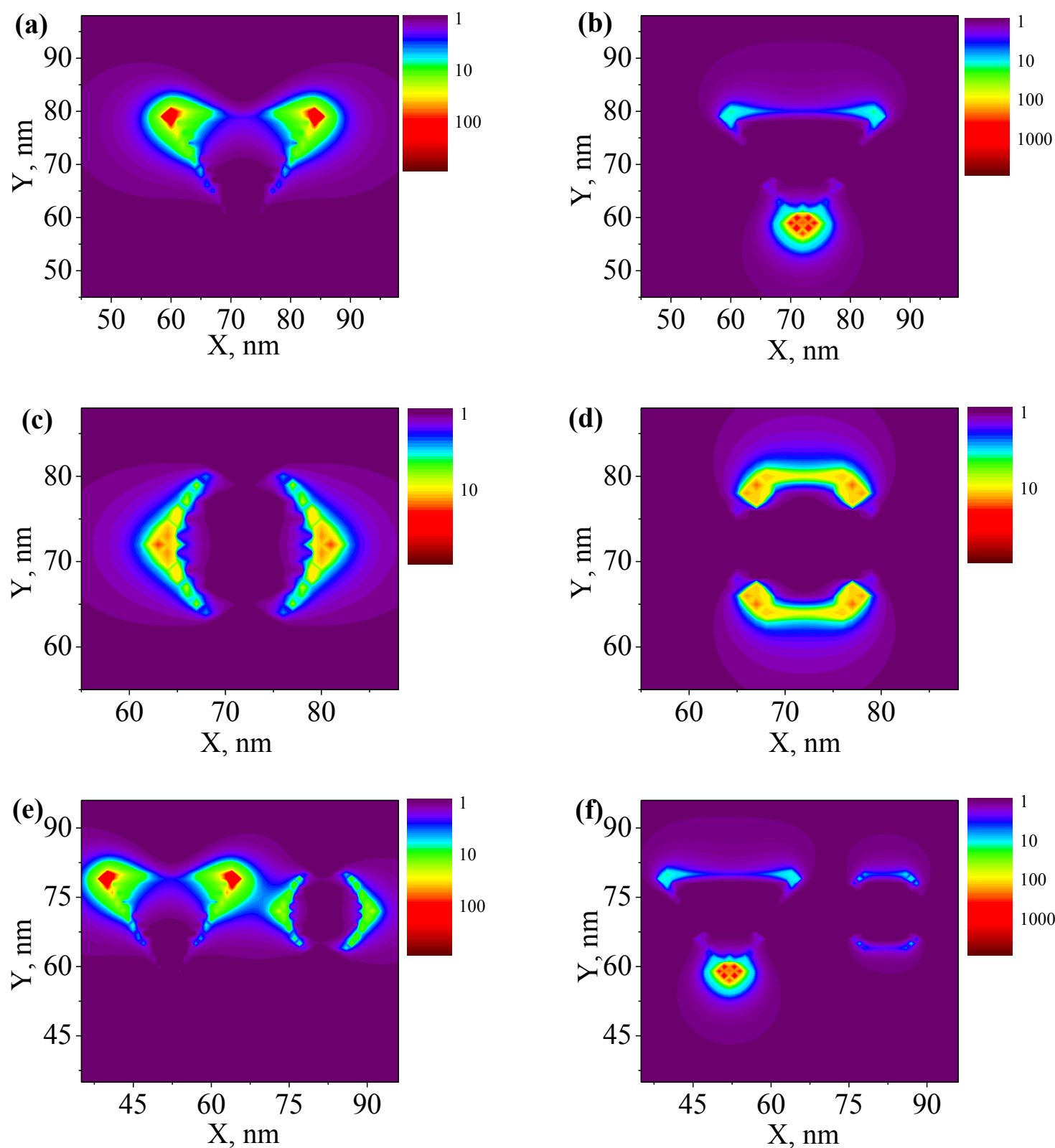

Fig. 5. The spatial electric field intensity distribution on the top bases of Au nanoprisms: (a), (b) trigonal nanoprism (model 1 in Fig. 3b); (c), (d) hexagonal nanoprism (model 2 in Fig. 3b); (e), (f) combination of trigonal and hexagonal nanoprisms (model 3 in Fig. $3 \mathrm{~b}$ ). All nanoprisms are located on the glass substrates and illuminated with orthogonal polarizations of the normally incident linearly-polarized light.

\section{Electric field distribution in the vicinity of the Au nanoprisms on glass substrates}

From the practical point of view, it is important to understand the relation between the geometrical parameters of plasmonic nanostructures and their performance as optical amplifiers, e.g., for application in surface-enhanced spectroscopy. To answer the question what shape and size of the abovementioned Au nanoprisms can provide higher signal enhancement, we calculated the electric field intensity distributions on the top bases of the investigated Au nanoprisms under the LSPR conditions using the FDTD method. Simulation results for the $\mathrm{Au}$ nanoprism models mentioned in Section 3 are presented in Figs 5 and 6. Calculations were performed for two orthogonal polarizations of the normally incident linearlypolarized light with the wavelength corresponding to the LSPR spectral position of the respective $\mathrm{Au}$ nanoprism model. 

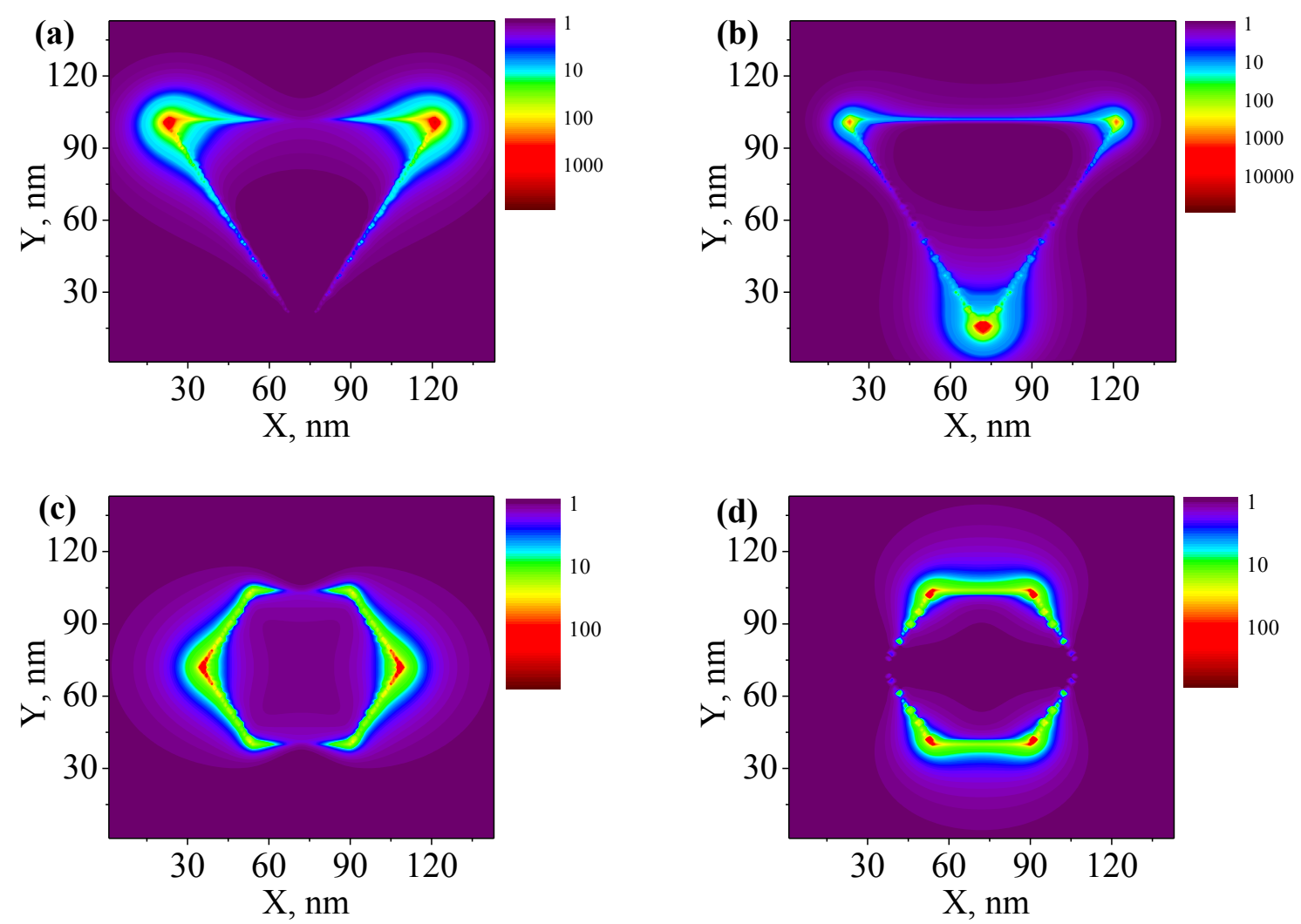

Fig. 6. The spatial electric field intensity distribution on the top bases of Au nanoprisms: (a), (b) trigonal nanoprism (model 1 in Fig. 3a); (c), (d) hexagonal nanoprism (model 2 in Fig. 3a). All nanoprisms are located on the glass substrates and illuminated with orthogonal polarizations of the normally incident linearly-polarized light.

As it can be seen from the obtained electric field intensity distributions (Fig. 5), there is a great enhancement of the electric field at the corners of the nanoprisms (from tens to thousands times, depending on the nanoprism shape and light polarization). If we compare the field enhancements at the corners of the trigonal and hexagonal nanoprisms, we shall see that it is about 10 times greater for the trigonal nanoprism than for the hexagonal one. This implies the opportunity to exploit the trigonal nanoprisms for surface-enhanced spectroscopy.

To examine how the size of the nanoparticles influences the enhancement of the electric field, the same simulation was performed for trigonal and hexagonal $\mathrm{Au}$ nanoprisms of larger size (model 1 and model 2 in Fig. 3a) (Fig. 6). From these results it is evident that while the size of the Au nanoprism increases from 25 to $100 \mathrm{~nm}$ (trigonal) and from 8 to $33 \mathrm{~nm}$ (hexagonal), the maximum field intensity enhancement increases approximately 10-fold.

In summary, the highest local electric field enhancement among the considered Au nanoprisms, up to 18000 times, was exhibited by the trigonal $\mathrm{Au}$ nanoprism with side dimension of $100 \mathrm{~nm}$, when they are illuminated with light polarized normally to one of the triangular base sides. To achieve the maximum performance and reproducibility of such nanostructures for surface-enhanced spectroscopy and LSPR sensing applications, they should be prepared as highly ordered nanoparticle arrays with uniform shape and size.

\section{Conclusions}

In conclusion, it was confirmed for the studied trigonal and hexagonal $\mathrm{Au}$ nanoprisms deposited on the glass substrates that the LSPR spectra depend on the nanocrystals shape and size. Experimentally obtained and theoretically modelled LSPR positions differ due to the high spread of the Au nanoprism shapes and sizes and aggregation of the nanocrystals. Local electric field intensity modeling under the LSPR conditions have demonstrated the promising potential of Au nanoprisms for surface-enhanced spectroscopy, with the highest enhancement provided by a $100 \mathrm{~nm}$ trigonal $\mathrm{Au}$ nanoprism on the glass substrate.

\section{Acknowledgements}

We are deeply indebted to "Melitek-Ukraine" for the possibility to carry out microscopy measurements. 


\section{References}

1. E. Hendry, T. Carpy, J. Johnston, M. Popland, R. Mikhaylovskiy, A. Lapthorn, S. Kelly, L. Barron, N. Gadegaard, M. Kadodwala, Ultrasensitive detection and characterization of biomolecules using superchiral fields // Nature Nanotechnology, 5, p. 783-787 (2010).

2. M. Abb, Yu. Wang, N. Papasimakis, C.H. de Groot, M. de Groot, L. Otto, Surface-enhanced infrared spectroscopy using metal oxide plasmonic antenna arrays // Nano Letters, 14(1), p. 346-352 (2013).

3. C. Leordean, B. Marta, A.-M. Gabudean, M. Focsan, I. Botiz, S. Astilean, Fabrication of highly active and cost effective SERS plasmonic substrates by electrophoretic deposition of gold nanoparticles on a DVD template // Appl. Surf. Sci. 349, p. 190-195 (2015).

4. T.I. Borodinova, V.G. Kravets, V.R. Romanyuk, Gold nanocrystals as a substrate for micro Raman spectroscopy // J. Nano- \& Electron. Phys. 4(2), p. 02039(7), (2012).

5. N.E. Motl, A.F. Smith, C.J. DeSantisa, S.E. Skrabalak, Engineering plasmonic metal colloids through composition and structural design // Chem. Soc. Rev. 43, p. 3823-3834 (2014).

6. A.M. Lopatynskyi, V.K. Lytvyn, V.I. Nazarenko, L.J. Guo, B.D. Lucas and V.I. Chegel, Au nanostructure arrays for plasmonic applications: annealed island films versus nanoimprint lithography // Nanoscale Res. Lett. 10, p. 99 (2015).

7. Sh. Zeng, X. Yu, W.-Ch. Law, Ya. Zhang, R. Hu, X-Q. Dinh, H.-P. Ho, K.-T. Yong, Size dependence of $\mathrm{Au}$ NP-enhanced surface plasmon resonance based on differential phase measurement // Sensors and Actuators B: Chemical, 176, p. 1128-1133 (2013).
8. A. Henry, J.M. Bingham, E. Ringe, L.D. Marks, G.C. Schatz, and R.P. Van Duyne, Correlated structure and optical property studies of plasmonic nanoparticles // The Journal of Physical Chemistry $C$, 115(19), p. 9291-9305 (2011).

9. Y.-Ch. Chang, Sh.-M. Wang, Hs.-Ch. Chung, Ch.-B. Tseng, and Sh.-H. Chang, Observation of absorption-dominated bonding dark plasmon mode from metal-insulator-metal nanodisk arrays fabricated by nanospherical-lens lithography // ACS Nano, 6(4), p. 3390-3396 (2012).

10. V. Juvé, M.F. Cardinal, A. Lombardi et al., Sizedependent surface plasmon resonance broadening in nonspherical nanoparticles: single gold nanorods // Nano Lett. 13(5), p. 2234-2240 (2013).

11. A. Ahmadivand, S. Golmohammadi, Plasmon resonance excitation and near field manipulating in gold nanopyramid arrangements at the telecommunication spectrum // J. Opt. Technol. 82(2), p. 6875 (2015).

12. A. Lopatynskyi, O. Lopatynska, L.J. Guo, V. Chegel, Localized surface plasmon resonance biosensor: theoretical study of sensitivity extended Mie approach. Part I // IEEE Sensors J. 11(2), p. 361-369 (2011).

13. F.J. Beck, E. Verhagen, S. Mokkapati, A. Polman, K.R. Catchpole, Resonant SPP modes supported by discrete metal nanoparticles on high-index substrates // Opt. Exp. 19(S2), p. A146-A156 (2011).

14. A.M. Kern, O.J.F. Martin, Modeling near-field properties of plasmonic nanoparticles: a surface integral approach // Proc. SPIE, 7395, p. 739518 (2009).

15. T.I. Borodinova, V.I. Sapsay, V.R. Romanyuk, Gold nanocrystals growth in the mixture of primary alcohols // J. Nano- \& Electron. Phys. 7(1), p. 01032(10) (2015). 